\title{
A colaboração entre escola, família e comunidade segundo Deslandes.
}

\section{The collaboration between school, family and community by Deslandes.}

\section{La collaboration entre l'école, famille et communauté selon Delandes.}

Élisabeth GRAVEL

\section{RESUMO}

Este depoimento trata da importância de Rollandes Deslandes, professora da Universidade do Quebec em Trois-Rivières no que diz respeito à relação escola- família e comunidade para o sucesso no aprendizado dos alunos.

Palavras- chave: escola, família, comunidade.

\section{ABSTRACT}

This text is about Rolland Deslandes'importance, professor at the University of Quebec in Trois-Rivieres, with respect to school-family, and community relationship a for success in student learning.

Index terms: school, family, community.

\section{RÉSUMÉ}

Cette temoignage souligne l'importance de Rolland Deslandes, professeur à l'Université du Québec à Trois-Rivières à l'égard de la relation école-famille et communauté à la réussite de l'apprentissage des élèves.

Mots clés: communauté scolaire, la famille. 
Dans l'une des écoles où je travaille, un comité se demande comment orienter le partenariat avec les parents pour les années à venir. On leur a demandé de se pencher sur la question. Aucun des membres du comité n'a déjà approfondi le sujet. Toutefois, ils savent que celui-ci est important, qu'un partenariat réussi peut avoir des impacts positifs sur les jeunes et sur le système scolaire. Ils désirent développer quelque chose d'efficace et de durable. Pour cela, ils veulent savoir ce qu'il est possible de faire. Ils aimeraient connaître les différentes sortes de partenariat et surtout, ceux qui donnent les meilleurs résultats. Ils savent aussi que j'ai quelques connaissances sur le sujet, donc je leur dresserai du mieux que je peux un portrait de l'évolution des relations parents-école et j'essaierai de les conseiller sur les façons de créer une réelle collaboration. Pour ce faire, je m'inspirerai beaucoup des recherches de Rollande Deslandes qui vont en ce sens.

Pour moi, la collaboration école-familles est très importante. J'y crois énormément et je sais que celle-ci peut avoir beaucoup d'impact positifs, tels augmenter le taux de réussite et le sentiment d'appartenance des élèves, diminuer le taux d'abandon scolaire, le taux d'absentéisme et les difficultés d'ordre comportementales (DESLANDES, 2001). C'est_donc un plaisir pour moi de participer à l'orientation des actions de l'école à ce niveau.

Premièrement, pour créer une sorte de déclencheur au travail du comité, je susciterais une discussion en questionnant les enseignantes sur la façon dont les choses se passaient quand eux, leurs parents ou leurs grandsparents allaient à l'école. Cela pourrait leur faire prendre conscience que bien des choses ont changé et qu'il va de soi que l'école prenne d'autres orientations pour maximiser leur partenariat avec les parents. Autrefois, dans les écoles, on travaillait beaucoup avec la peur et la soumission et l'école n'avait qu'une mission : instruire. En milieux ruraux, l'importance de l'école était moindre et les relations parents-école presque inexistantes. 
Les attentes des parents envers l'école étaient surtout tournées vers les apprentissages de base comme lire, écrire et compter. Si l'enfant réussissait bien, tant mieux. Sinon, il pouvait se débrouiller et travailler quand même. Il n'avait pas besoin d'un diplôme pour trouver un emploi. Or, la collaboration école-famille n'était pas un souci. Peu de parents s'impliquaient dans la vie scolaire. Ils avaient beaucoup trop à faire à la maison étant donné les familles nombreuses. Depuis ce temps, plusieurs changements sont survenus dans la société, ce qui a eu un impact sur les relations entre l'école et la famille. Cela donné lieu à différentes théories sur la collaboration écolefamille.

Selon moi, il serait important que les enseignantes du comité sojent au courant des différentes théories existantes sur la collaboration écolefamille, afin de pouvoir préciser leurs intentions. Voici un bref résumé des différents modèles, tiré des travaux de Deslandes (1996) :

1) Le modèle de l'influence distincte

Ce modèle soutient que l'école et la famille sont efficaces lorsque les agents éducatifs poursuivent des objectifs et effectuent des activités en parallèle (Epstein, 1990; Parson, 1959; Waller, 1932; Weber, 1947). Selon cette perspective, les deux institutions, la famille et l'école, sont considérés comme deux mondes à part (Lightfood, 1978), deux influences distinctes. Les enseignants ne contactent les parents que si l'élève présente de sérieux problèmes de comportement ou d'apprentissage et vice versa (Epstein, 1990).

2) Le modèle de l'influence séquentielle

Ce modèle identifie une séquence de stades critiques au cours desquels la famille et l'école contribuent, chacune à son tour, au développement de l'enfant et à son éducation (Epstein, 1987, 1990, 1992). Un des principes à la base de ce modèle est que les premières années de la vie déterminent le succès futur de l'enfant et que les parents ont la responsabilité des premières phases critiques de l'apprentissage qui 
préparent l'enfant à l'entrée scolaire. Par la suite, les enseignants assument les principales responsabilités en ce qui concerne l'éducation de l'enfant d'âge scolaire (Bloom, 1964; Epstein, 1987, 1990, 1992; Freud, 1938; Piaget et Inhelder, 1969)

Selon moi, ces deux premiers modèles traduisent la façon dont on voyait les choses au début du siècle. Avec toutes les recherches qui ont été faites aujourd'hui, on ne peut plus considérer l'école et la maison comme deux monde à part ou deux influences distinctes. Pour le bien du jeune et son besoin de constance et de cohérence, les deux parties doivent faire consensus. À mon avis, les deux modèles qui suivent se rapprochent davantage des croyances actuelles.

3) Le modèle de l'influence intégrée

Celui-ci se fonde sur un modèle écologique représenté par un ensemble de cercles concentriques d'interaction et d'influence entre les individus et les nombreux environnements auxquels ils appartiennent (Bronfenbrenner, 1979).

4) Le modèle de l'influence partagée

Ce modèle privilégie la coopération et la complémentarité entre l'école et la famille (Epstein, 1987, 1990, 1992). Il s'inscrit dans une optique de prévention des conflits, nécessitant des interactions continuelles entre les parents et les enseignants pour développer et maintenir des relations positives (Epstein 1990). Le modèle représente aussi la continuité dans les interactions entre l'école et les familles au fil des ans et les changements dans la participation parentale au suivi scolaire en fonction de l'âge des élèves et de leur stade de développement. Ce modèle est illustré par des sphères représentant la famille et l'école qui peuvent être poussées l'une vers l'autre ou encore éloignées l'une de l'autre par l'énergie, le comportement ou les actions de chacun des deux milieux.

Suite à la présentation de ces modèles, je demanderais aux enseignantes comment elles envisagent les relations école-famille, je leur 
demanderais de se positionner. À mon avis, pour orienter leurs actions, elles devraient s'imprégner des principes des deux derniers modèles. Ceux-ci font ressortir l'importance des deux influences (famille et école) sur le développement de l'enfant. Ils misent sur le travail d'équipe et la complémentarité des deux partis, choses en lesquelles je crois également.

Afin d'agir de façon efficace, je présenterais brièvement aux enseignantes la recherche-action menée par Deslandes. Même si celle-ci n'a pas donné les résultats escomptés, un constat important en ressort; celui que la collaboration école-famille doit dépasser le stade du «projet » et devenir une préoccupation permanente de chaque école. Toujours selon la recherche-action de Deslandes, pour qu'un véritable partenariat s'établisse, il faut avoir des visées de changement de mentalités et d'attitudes, qui ne peuvent s'opérer qu'à long terme. Les membres du comité doivent dong être conscients qu'ils devront faire preuve de persévérance et de rigueur. Un réel partenariat ne s'instaure pas en quelques mois.

De plus, je suggérerais aussi aux enseignantes faisant partie du comité d'utiliser le document «Rapprocher l'école et les familles », du Ministere de l'éducation du Québec (MELS). Celui-ci propose des questionnaires destinés aux membres du personnel de l'école et aux parents. Il serait certainement important, s'ils veulent poser des actions durables que les membres du comité s'intéressent au point de vue de tous. En effet, je crois que la collaboration école-famille, ce n'est pas l'affaire de 3 ou 4 personnes dans l'école. Il faut que tous les acteurs se mobilisent et y croient. Il s'avérerait donc pertinent d'utiliser l'outil «Inventaire des pratiques de l'école» proposé par le MELS afin d'impliquer tout le personnel et de se donner une vue d'ensemble de l'état de la collaboration avec les parents en relation avec les quatre volets (diversifier la communication, faciliter l'exercice du rôle de parent, encourager la participation des parents à la vie de l'école, collaborer plus étroitement avec la communauté pour répondre aux besoins des familles). Les enseignantes du comité devraient aussi 
utiliser le «Questionnaire destiné aux parents » proposé par le MELS afin de permettre aux parents de donner leur point de vue, leurs commentaires et leurs suggestions et d'exprimer leurs besoins sur le ou les volets retenus par l'équipe-école. À la suite de la passation des outils, les membres du comité pourront analyser les résultats et par la suite planifier les interventions. Le document du MELS explique très bien les modalités de passation des instruments et les quatre domaines privilégiés de collaboration entre l'école et la famille. Tous les membres du comité devraient en prendre connaissance et se l'approprier.

Bien sûr, le comité pourrait proposer aux enseignants de l'école certaines choses et mettre en place différentes actions rapidement (offrir des conférences ou des ateliers aux parents, préparer des dépliants ou des guides pour informer les parents de ce qui se passe à l'école, bonifier les moyens de communication, etc.). Toutefois, s'ils veulent que leurs actions répondent vraiment à un besoin, qu'elles soient efficaces et durables et adaptées à leur milieu, il est selon moi essentiel qu'ils prennent le temps pour bien cerner la situation et planifier les interventions - ce que les outils proposés par le document «Rapprocher les familles » permettent de faire.

Finalement, je crois que ces étapes permettraient au comité de bien entreprendre leur travail. Ils auraient une idée générale de l'évolution des relations école-famille et des différents types de collaboration, ce qui contribuerait à augmenter leurs connaissances, donc leur sentiment de compétence par rapport au sujet qu'ils veulent développer. Ce qui les inciterait sans doute à s'impliquer davantage. De plus, ils auraient en main des outils pour planifier des actions efficaces. À mon avis, il n'y a pas de formule magique pour créer une collaboration ou un partenariat réel et idéal. Les idées doivent être amenées par le milieu, selon les besoins, les demandes, la créativité et les valeurs des gens concerné. 


\section{RÉFÉRENCES BIBLIOGRAPHIQUES}

MINISTERE DE L'ÉDUCATION DU QUÉBEC (2004). Rapprocher les familles et l'école primaire. Guide d'utilisation de deux instruments à l'intention des écoles primaires. Gouvernement du Québec.

DESLANDES, R.. Collaboration école-famille: defies sociaux et scolaires. In: Options CSQ Hors Serie, n.1. p. 145-167, 2006.

\section{Autora:}

Élisabeth GRAVEL

Université du Québec à Chicoutimi

Contato: Elisabeth.gravel@uqac.ca

Texto recebido em março de 2008.

Texto aprovado para publicação em maio de 2008.

\section{Como citar este texto:}

GRAVEL, E.. La collaboration entre l'école, famille et communatuté selon Delandes. Revista Acolhendo a Alfabetização nos Países de Língua Portuguesa, Brasil, São Paulø, volume 1, no. 11, pp. 177 - 183, Set.. 2011. Disponível em: <http://www.acoalfaplp.net>. 